\title{
Linking long non-coding RNAs and SWI/ SNF complexes to chromatin remodeling in cancer
}

\author{
Yanyan Tang ${ }^{1,2,3}$, Jinpeng Wang ${ }^{1,2}$, Yu Lian ${ }^{1,2}$, Chunmei Fan², Ping Zhang ${ }^{4}$, Yingfen $\mathrm{Wu}^{2}$, Xiayu Li ${ }^{2,3}$, Fang Xiong ${ }^{1}$, \\ Xiaoling Li $\mathrm{Li}^{1,2,3}$, Guiyuan $\mathrm{Li}^{1,2,3}$, Wei Xiong ${ }^{1,2,3^{*}}$ and Zhaoyang Zeng ${ }^{1,2,3^{*}}$
}

\begin{abstract}
Chromatin remodeling controls gene expression and signaling pathway activation, and aberrant chromatin structure and gene dysregulation are primary characteristics of human cancer progression. Recent reports have shown that long non-coding RNAs (IncRNAs) are tightly associated with chromatin remodeling. In this review, we focused on important chromatin remodelers called the switching defective/sucrose nonfermenting (SWI/SNF) complexes, which use the energy of ATP hydrolysis to control gene transcription by altering chromatin structure. We summarize a link between IncRNAs and the SWI/SNF complexes and their role in chromatin remodeling and gene expression regulation in cancer, thereby providing systematic information and a better understanding of carcinogenesis.
\end{abstract}

Keywords: Long non-coding RNAs (IncRNAs), The SWI/SNF complexes, Chromatin remodeling, Gene expression, Carcinogenesis

\section{Background}

Cancer is one of the leading causes of death in the world. Dysregulation of chromatin remodeling and cytoskeleton organization is tightly associated with the progression of cancer [1]. Chromatin remodeling is the dynamic modification of the chromatin architecture to allow regulatory transcription machinery proteins access to condensed genomic DNA and thereby control gene expression [2, 3]. Some specific protein complexes, which can move, eject, replace or restructure nucleosomes with energy from the hydrolysis of adenosine triphosphate (ATP), play a pivotal role in the process of chromatin remodeling by providing proper nucleosome position and density [4]. Two major classes of protein complexes control the process of chromatin remodeling. One class is the covalent histone-modifying complexes, and the other is the ATPdependent chromatin remodeling complexes. Chromatin remodeling is currently a major therapeutic strategy in the treatment of several cancers.

\footnotetext{
* Correspondence: xiongwei@csu.edu.cn; zengzhaoyang@csu.edu.cn 'The Key Laboratory of Carcinogenesis of the Chinese Ministry of Health, Xiangya Hospital, Central South University, Changsha, Hunan, China Full list of author information is available at the end of the article
}

In eukaryotes, according to the different ATP enzyme and protein subunits, the ATP-dependent chromatin remodeling complexes can be divided into four classes: switching defective/sucrose nonfermenting (SWI/SNF), imitation switch (ISWI), chromodomain helicase DNA binding (CHD), and inositol requiring 80 (INO80) [2]. There are large differences in the mechanisms of the four classes. The SWI/SNF complexes recognize the nucleosome and naked DNA with a high affinity and can move the nucleosome by interacting with DNA, resulting in a more easily affected DNA region [5]. SWI/SNF is usually associated with activated chromatin and can silence gene expression. ISWI recognizes only the nucleosome to silence chromatin with low activity. Some CHD complexes slide or eject nucleosomes to promote transcription, and others play repressive roles with histone deacetylases. INO80 can activate transcription and DNA repair [2].

Long non-coding RNAs (lncRNAs) are a subset of noncoding RNAs, which exceed 200 nucleotides in length [6-8]. Accumulating evidence has confirmed that lncRNAs contribute to cancer initiation and progression through regulating gene transcription and post-transcriptional regulation through chromatin remodeling [9-16]. In this review, we 
described the relationship between lncRNAs and the SWI/ SNF complexes on the mechanism of transcriptional regulation and chromatin modification in cancer. This link will provide new insights for future studies on cancer.

\section{The structure and composition of the SWI/SNF complexes}

The SWI/SNF complexes, which were first discovered in yeast, regulate the expression of homothallic switching endonuclease $(\mathrm{HO})$ and the transforming enzyme sucrose invertase (SUC2) [17]. Among these complexes, $\mathrm{HO}$ is a mating type switch (SWI) and SUC2 is necessary for sucrose non-fermentation (SNF) $[18,19]$. Therefore, the complexes have come to be known as the SWI/SNF complexes.

The SWI/SNF complexes contain a conserved DNAdependent ATPase as their catalytic subunit and distinct flanking domains, such as helicase-SANT-associated domains (HSA) and bromodomain proteins [3]. The ATPase domain (core subunit) is composed of two parts: DEXDc and HELICc. DEXDc, also called SNF2-N, contains an ATP $\mathrm{Mg}^{2+}$ binding site and a motor unit called the DEAD box domain, which converts ATP energy to mechanical movement [20]. HSA can bind to ARPs and actin [2]. At the C-terminal, a bromodomain is located, which can recognize acetylated lysines in histones and contributes to increasing remodeling efficiency [2] (Fig. 1a).

Most remodeling enzymes form a multi-subunit complex to perform the remodeling function. The SWI/SNF complexes are divided into two sub-classes as distinguished by different subunits; one sub-class is the BRG/ hBRM associated factors (BAF) complexes, and the other is the polybromo associated BAF (PBAF) complexes [3]. The two sub-classes of complexes are always composed of 8 to 14 subunits, including several core subunits (ATPase subunit), the BAF complex containing either Brahma (BRM) or BRM-related Gene 1 (BRG1), and the PBAF complex only containing BRG1 [21]. The other core components are BAF155 (SMARCC1 gene encoded), BAF170 (SMARCC2) and SNF5/BAF47/INI1 (SMARCB1) in both the BAF and PBAF complexes. The core subunits are the most important subunits, possessing the same level of remodeling activity as the entire SWI/SNF complex [22, 23]. Core subunits are mostly involved in double-strand break (DSB) repair and nucleotide excision repair.

In addition to these core subunits, the signature subunits of the SWI/SNF complexes, which are associated with elongation and transcription, are BAF250A/B (ARID1A/B) in BAF complexes and BAF180 (PBRM1), BAF200 (ARID2) and BRD7 in PBAF complexes [4, 24]. Moreover, many accessory subunits are part of the SWI/SNF complexes, such as BAF57, BAF53A/B, BAF60A/B/C and $\beta$-actin. The function of each subunit is slightly different through conserved proteins, and unique attendant subunits distinguish each complex (Fig. 1b and c).

\section{The SWI/SNF complexes mediates chromatin remodeling}

The hypothesized steps of chromatin remodeling include the recruitment and localization of the SWI/SNF complexes to target genes followed by the hydrolysis of ATP to release energy and alter chromatin structure. Early observations showed that there are three models of the SWI/SNF complexes recruited and located on chromatin [25]. The first is the "non-targeting model", meaning that the SWI/SNF complexes locate anywhere on chromatin in a random manner, requiring only one DNA binding transcription factor. In this model, chromatin structure remodeling will occur, but this model seems inadequate to clarify the function of the SWI/SNF complexes [25]. The second model is "RNA polymerase II association", in which the SWI/SNF complexes are associated with a specific nucleosome by RNA polymerase II [26]. The last model is "targeting by activators", in which the SWI/SNF complexes are recruited to target genes by transcriptional activators and transcription factors [27].

There are two related models for the mechanism by which the SWI/SNF complexes remodel chromatin conformations. The "nucleosome slide model", where the SWI/ SNF complexes use the energy of ATP-hydrolysis to remodel histones, which results in changes in the chromatin structure. The SWI/SNF complexes can work as a DNA translocase enzyme. When the DNA double strand is opened, the SWI/SNF complexes can make the nucleosome slide along the DNA. In this way, the relative movement of histones and the DNA change the position of the nucleosome. At the same time, restricted enzyme sites of DNA are exposed, which results in transcription factors being bound to corresponding elements [28]. However, the slide model changes only the position of the nucleosome, which cannot explain how a large amount of exposed DNA can be formed in a closely packed region. Therefore, there may be some other mechanisms for chromatin remodeling. The other model is the "bulge model", in which the SWI/ SNF complexes push or pull linker DNA into nucleosome regions, resulting in the DNA bulge exposure or inhibition of a section of DNA sequence. No matter the assumptions, the DNA bulge will change the interaction of the histone octamer with the DNA, and the relative position of DNA on a histone will change and cause the sliding of the nucleosome on DNA. Eventually, chromatin remodeling mediated by the SWI/SNF complexes will cause activation or inhibition of the corresponding genes [28].

\section{LncRNAs interact with the SWI/SNF complexes regulating chromatin remodeling in cancer}

The chromatinic structure can undergo dynamic changes in the cell. When the chromatic structure is compact, it will prevent transcription factors and RNA polymerase recruitment and binding to specific DNA 
a

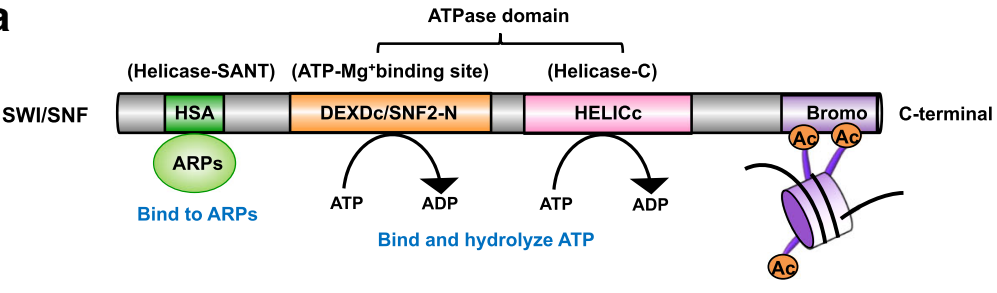

b

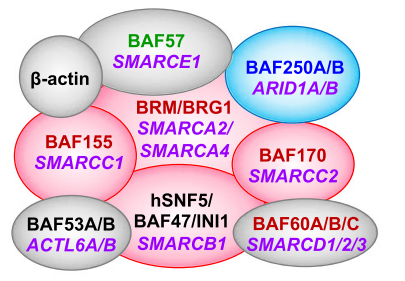

BAF complex

Bind to acetylated lysines in histones

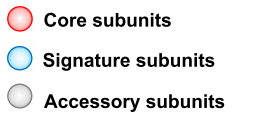

DSB repair and nucleotide excision repair

Elongation and transcription

Cell-cycle, proliferation, differentiation and

stem cell self-renewal

C

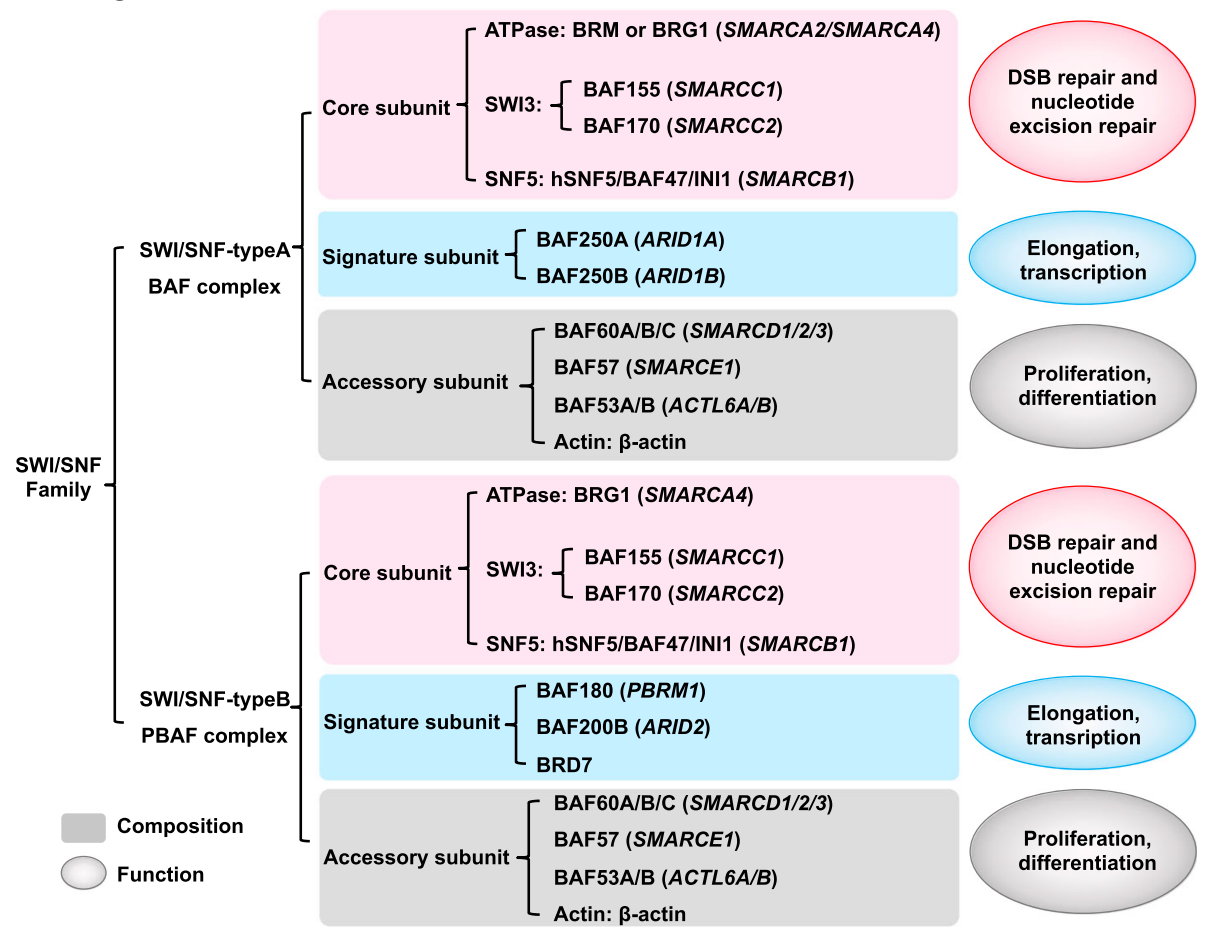

Fig. 1 The structure and composition of the SWI/SNF complexes in human. a The structure of the SWI/SNF complexes. The SWI/SNF complexes contain a DNA-dependent ATPase as its catalytic subunit and distinct flanking domains, such as HSA and bromodomain. $\mathbf{b}$ The subfamily of the SWI/SNF complexes. The SWI/SNF complexes consist of several core subunits (pink), signature subunits (blue) and additional accessory subunits (gray). The subunit coded gene is marked in purple with italics. Different colors of characters in a complex indicate the function of various subunits. $\mathbf{c}$ The outline of the SWI/SNF complexes. The SWI/SNF complexes are divided into two types: BAF and PBAF. Two types of the SWI/SNF complexes contain different subunits, which are related to distinct functions 
sequences, leaving genes silenced. While the chromatinic structure is loose, transcription factors can bind to the promoter of a specific gene and activate transcription. The SWI/SNF complexes usually remodel chromatin in a loose state and are associated with the activation or suppression of gene expression [29]. SWI/SNF affects genes expression through chromatin status, such as nucleosome positioning, exchanging or moving [30, 31]. Recent studies showed that lncRNAs regulate chromatin modification and gene expression through their interactions with the SWI/SNF complexes. According to many reports, the interaction between IncRNAs and the SWI/ SNF complexes can be divided into two models in cancer: the binding model and the recruiting model.

The binding model states that lncRNAs can directly bind to the subunit of the SWI/SNF complexes and serve as a guide to anchor the SWI/SNF complexes or function as a decoy to keep chromatin modifiers away from specific genomic sites. Moreover, lncRNAs can be incorporated into the SWI/SNF complexes and function as a scaffold to assemble the complex for chromatin remodeling [32]. One such lncRNA is called Second Chromosome Locus Associated with Prostate 1 (SChLAP1), which is overexpressed in a subset of prostate cancers. The mechanism of SChLAP1 is direct binding to hSNF5, which can antagonize tumor suppressive functions of the SWI/SNF complexes by decreasing their genomic binding. Therefore, SChLAP1 promotes tumor cell invasion and metastasis by binding and impairing proper SWI/SNF regulation of gene expression [33, 34] (Fig. 2). The IncRNA UCA1 can bind to BRG1, the core subunit of the SWI/SNF complexes, and damage the remodeling activity of BRG1, which reduces the expression of $\mathrm{p} 21$ by blocking BRG1 binding to the p21 promoter and promotes the proliferation of bladder cancer cells [35]. Nuclear paraspeckle assembly transcript 1 (NEAT1) is a nuclear-restricted lncRNA, which is dysregulated in various human cancers, including leukemia, bladder cancer, lung cancer, breast cancer and gastric cancer [36-40]. In the process of IncRNA-dependent nuclear body assembly, NEAT1 directly interacts with the SWI/SNF core unit, BRG1 or BRM, to form the paraspeckle structure, which leads to cell cycle arrest and affects cancer progression [41, 42]. The lncRNA Evf2 can directly bind to BRG1 through distinct binding sites and repress the activity of BRG1 ATPase and chromatin remodeling [43]. The lncRNA HIF1A-AS1 binds to BRG1, which contributes to the regulation of cell proliferation and apoptosis in cancer [44, 45] (Fig. 2).

The recruiting model involves IncRNAs recruited SWI/SNF complexes or some core subunits to the target gene, thereby affecting the gene structure and expression. For instance, the lncRNA $\operatorname{lncTCF} 7$ was overexpressed in hepatocellular carcinoma and promoted tumorigenesis of liver cancer stem cells. The mechanism is $\operatorname{lncTCF7}$ recruitment of the core subunits of the SWI/ SNF complexes, BRG1, SNF5 and BAF170, to the TCF7 promoter. Furthermore, the SWI/SNF complexes trigger the TCF7 gene and promote tumor progression [46]. Another example of the recruiting model is RNA Polymerase V-generated lncRNA, which can guide the SWI/ SNF complexes to genome specific loci [47]. These above lncRNAs show that lncRNAs regulate the carcinogenesis of cancer through interacting with different subunits of the SWI/SNF complexes in two different manners (Table 1 and Fig. 2).

Furthermore, IncRNAs interact with the SWI/SNF complexes to affect tumorigenesis and development in different ways. For instance, a Wnt signaling pathway activating non-coding RNA $\operatorname{lncTCF7}$, which recruits SWI/ SNF to the promoter of TCF7, activates the Wnt signaling pathway by upregulating the expression of TCF7, which then promotes self-renewal of liver tumor stem cells [46]. LncRNAs can also recruit inflammatory transcription factor assembly into the SWI/SNF complexes. LincRNA Cox2 promotes the transcription of late inflammatory genes in macrophages by regulating SWI/ SNF mediated chromatin remodeling. Under the stimulation of bacterial LPS, lincRNA Cox2 is required for the transcription of the late inflammatory response genes that regulate NF-kB. Specifically, lincRNA Cox2 is assembled into the SWI/SNF complexes in cells after LPS stimulation, which causes the lincRNA Cox2-SWI/SNF complex to modulate the NF- $\mathrm{kB}$ subunits assembling into the SWI/SNF complexes. Therefore, SWI/SNF-related chromatin remodeling occurs in macrophages and causes the transcriptional regulation of the late response genes in the innate immune cells [48]. The immune system can regulate tumor progression and is one of the important mechanisms in tumorigenesis; because of this close relationship between the immune system and tumors, we hypothesize that there are many other kinds of lncRNAs that could assemble to SWI/SNF to recruit transcription factors, thereby affecting the progression of tumors and showing some form of inflammation regulation.

Some oncogenes also participate in the regulation of the SWI/SNF complexes in cancer. $M Y C$, a multifunctional oncogene, plays a critical role in cell proliferation, differentiation, apoptosis, and genetic instability [49]. It can interact with different subunits of SWI/SNF. For example, the core subunit of SWI/SNF SNF5/INI1 interacts with $M Y C$ through the $M Y C$ basic helix loop helix (bHLH), leucine zipper (Zip) and the INI1 repeat 1 (Rpt1) domains and contributes to the transcription of MYC target genes [50]. BAF250A, a subunit of the SWI/ SNF complexes, directly inhibits the expression of $M Y C$ in differentiating cells [51]. Methylated BAF155 (the core subunit of SWI/SNF complexes) can also be recruited to the $M Y C$ target gene GADD45A and promote cancer 


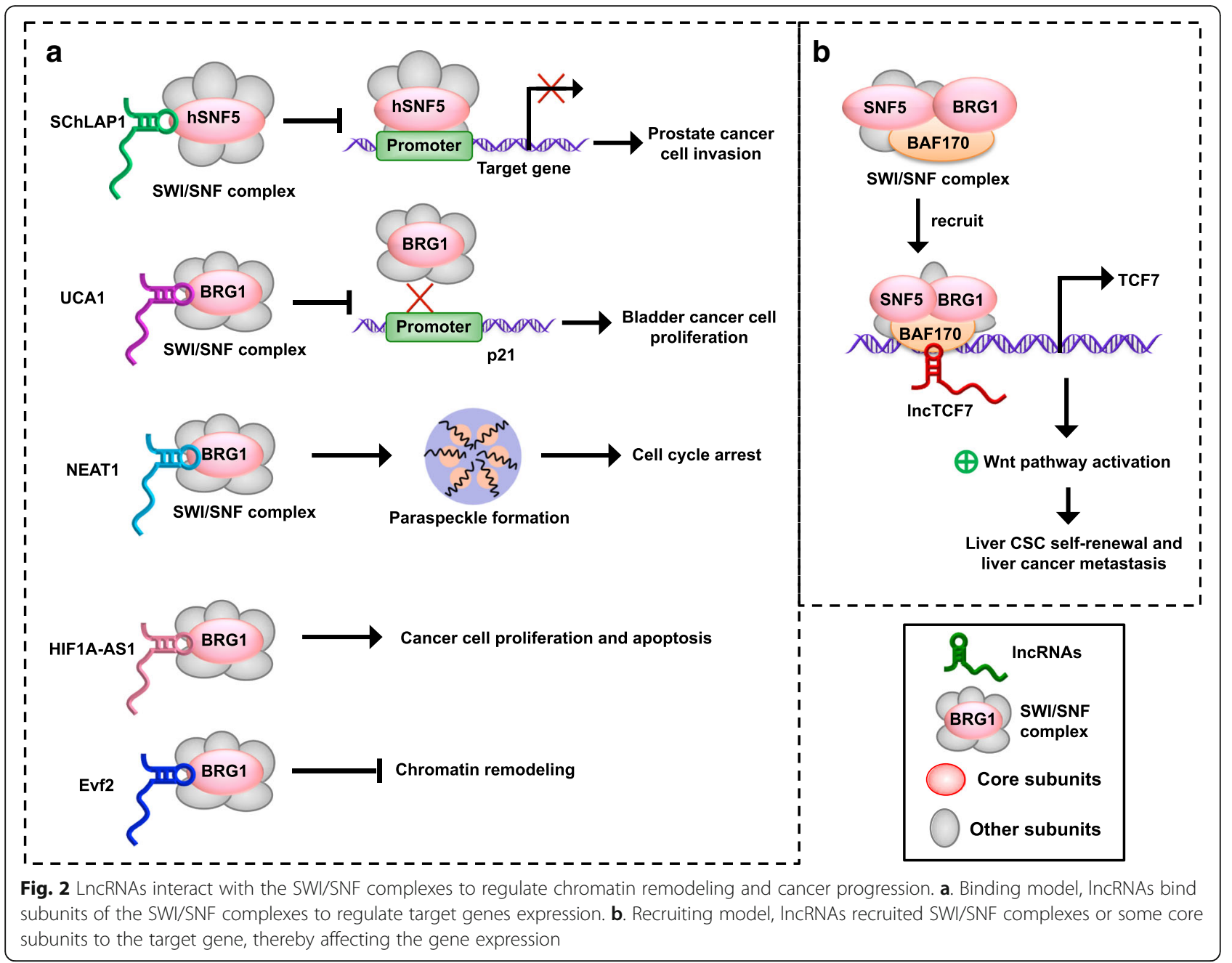

progression [49]. BRG1 is a tumor suppressor in most cancer types, but it promotes $M Y C$ transcription and maintains oncogenic programming in leukemia cells [52]. The transcriptional activation of $M Y C$ requires the interaction with the $M Y C$-associated factor $\mathrm{X}$ gene (MAX), to form the MYC-MAX dimer [53]. The SWI/ SNF complexes can also interact with $M A X$, for instance, BRG1 directly recruits to the $M A X$ promoter and regulates the expression of $M A X$ in lung cancer [54]. These studies provide evidence that an interaction between the SWI/SNF complexes and MYC is essential in cancer.

$K R A S$, first discovered in the rat sarcoma virus, plays a critical role in human cancer [55]. Knockdown of SWI/ SNF subunits BRM, BRG1, hSNF5 and BAF250A decrease the activity of KRAS in colon cancer cells [56].

Table 1 Summary of IncRNAs interacting with the SWI/SNF complex in cancer

\begin{tabular}{|c|c|c|c|}
\hline LncRNAs & Interaction of IncRNA with SWI/SNF & Cancer type & Refs \\
\hline SChLAP1 & $\begin{array}{l}\text { SChLAP1 binds to hSNF5/BAF47 and antagonizes the tumor } \\
\text { suppressive functions of the SWI/SNF complex. }\end{array}$ & Prostate cancer & {$[33,34]$} \\
\hline LnCTCF7 & $\begin{array}{l}\text { LnCTCF7 recruits BRG1, SNF5 and BAF170 to the TCF promoter and } \\
\text { activates the Wht signaling pathway. }\end{array}$ & Liver cancer & [46] \\
\hline NEAT1 & NEAT1 interacts with BRG1 or BRM to form the paraspeckle structure. & Various human cancers & {$[41,42]$} \\
\hline UCA1 & $\begin{array}{l}\text { UCA1 binds and represses the chromatin remodeling activity of BRG1, } \\
\text { which promotes bladder cancer cell proliferation. }\end{array}$ & Bladder cancer & [35] \\
\hline HIFIA-ASI & $\begin{array}{l}\text { HIF1A-AS1 interacts with BRG1, which contributes to regulate cell } \\
\text { proliferation and apoptosis. }\end{array}$ & Lung cancer & {$[44,45]$} \\
\hline Evf2 & Evf2 represses the activity of BRG1 ATPase and chromatin remodeling. & Unknown & [43] \\
\hline
\end{tabular}


Table 2 The SWI/SNF complex affects cancer development by regulating oncogene expression

\begin{tabular}{|c|c|c|c|}
\hline SWI/SNF & Interaction of SWI/SNF with oncogene & Cancer types/Phenotypes & Refs \\
\hline BRG1 & $\begin{array}{l}\text { BRG1 promotes myc transcription and maintenance of oncogenic } \\
\text { programming. }\end{array}$ & Leukemia & [52] \\
\hline BRG1 & $\begin{array}{l}\text { BRG1 directly recruits to the MAX (myc-associated factor } X \text { gene) } \\
\text { promoter and regulates the expression of MAX. }\end{array}$ & Lung cancer & [54] \\
\hline SNF5/INI1 & $\begin{array}{l}\text { SNF5/INI } 1 \text { interacts with c-myc and recruits the SWI/SNF complex, } \\
\text { which contributes to the transcription of MYC target genes. }\end{array}$ & Apoptosis & [50] \\
\hline BAF250A & BAF250A subunit directly inhibits the expression of MYC. & Differentiation associated cell cycle arrest & [51] \\
\hline BAF155 & Methylated BAF155 recruited to MYC target gene, GADD45A. & Breast cancer progression and metastasis & [49] \\
\hline BRG1 & $\begin{array}{l}\text { BRG1 inactivating mutation may cooperate with KRAS mutation } \\
\text { during carcinogenesis. }\end{array}$ & Lung Cancer & [57] \\
\hline BRM, BRG1, hSNF5, BAF250A & $\begin{array}{l}\text { The RAS inhibitor RasGAP1 can inhibit the BRM, knockdown } \\
\text { SWI/SNF members BRM, BRG1, hSNF5 and BAF250A, } \\
\text { and decrease the active KRAS }\end{array}$ & Colon cancer cell & [56] \\
\hline BRG1 & $\begin{array}{l}\text { BRG1 inactivating mutation cooperates with oncogenic } \\
\text { KRAS and promote the progression of pancreatic ductal } \\
\text { adenocarcinoma. }\end{array}$ & Pancreatic ductal adenocarcinoma & [59] \\
\hline SNF5 & $\begin{array}{l}\text { SNF5 can bind and activate the tumor repressor INK4A/ARF, } \\
\text { which is in response to oncogene KRAS, indicating that the } \\
\text { function of SWI/SNF link to oncogene KRAS. }\end{array}$ & Lung tumor & [58] \\
\hline
\end{tabular}

The Ras inhibitor RasGAP1 can also inhibit the BRM gene. BRG1 inactivating mutations may cooperate with KRAS mutations during carcinogenesis [57]. SNF5 can bind and activate the tumor repressor INK4A/ARF, which is in response to the oncogene KRAS [58]. Table 2 lists the various subunits of the SWI/SNF complexes associated with oncogenes such as MYC and KRAS in different cancer types. Understanding these relationships may provide insights into human genomic disorders, cell migration and cancer metastasis.

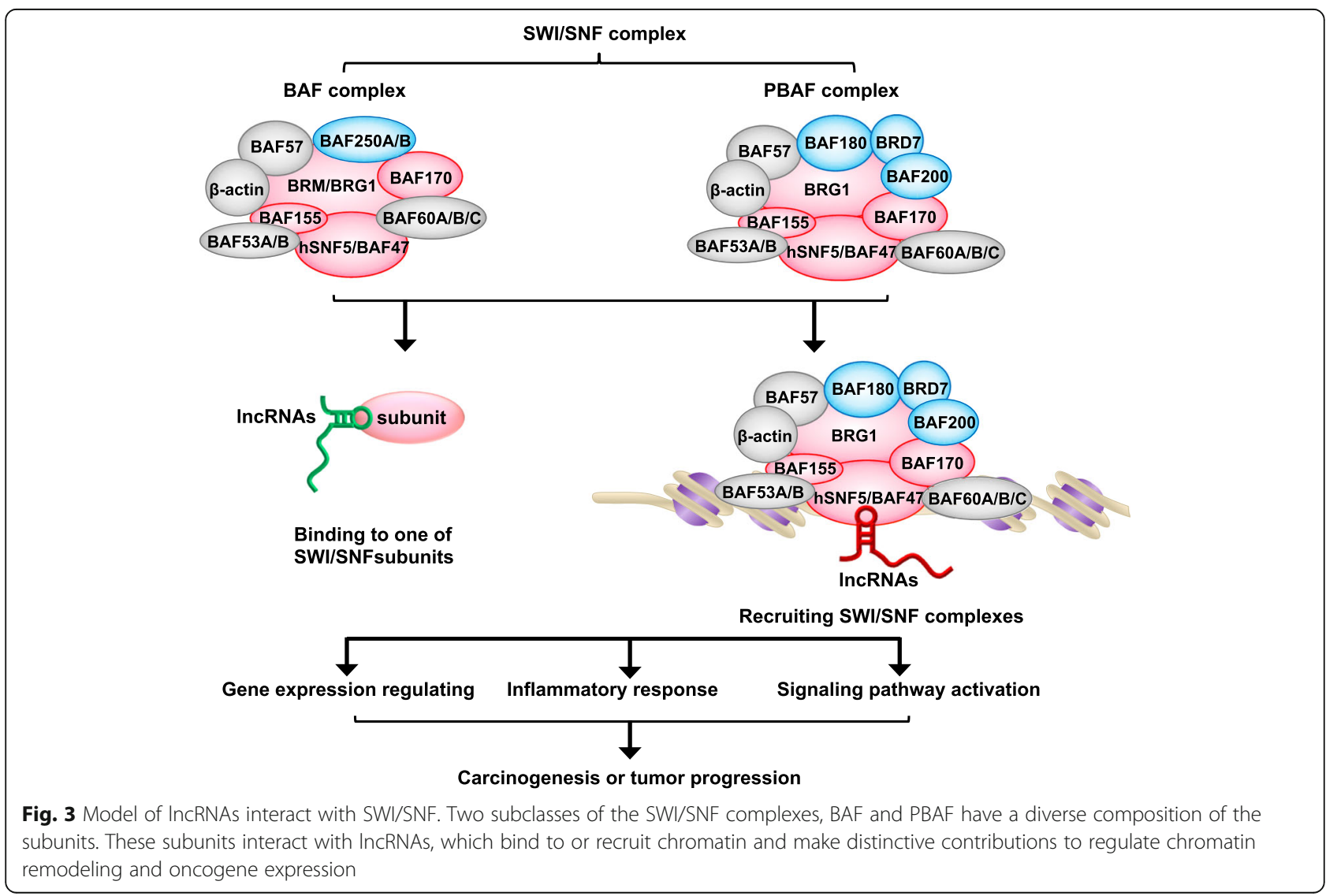




\section{Conclusion}

In this review, we have summarized different subunits of the SWI/SNF complexes. These subunits recruit to chromatin for chromatin remodeling and transcriptional control through interactions with lncRNAs (Fig. 3). This review gave a detailed description of the function of the SWI/SNF complexes and the relationship between the SWI/SNF complexes and lncRNAs, which will offer new insights into the processes of cancer and provide novel therapeutic approaches to SWI/SNF-mutant cancers.

\begin{abstract}
Abbreviations
ARPS: Actin-related protein; ATP: Adenosine triphosphate; BAF: BRG/hBRM associated factors; bHLH: Basic helix loop helix; BRG1: BRM related gene 1 BRM: Brahma; Bromo: Bromodomain; CHD: Chromodomain helicase DNA binding; DSB: DNA double strand break; $\mathrm{HO}$ : Homothallic switching endonuclease; INO80: Inositol requiring 80; ISWI: Imitation switch; LncRNAs: Long noncoding RNAs; MAX: Myc-associated factor X gene; NEAT1: Nuclear paraspeckle assembly transcript 1; NSCLC: Non-small lung cancers; PBAF: Polybromo associated BAF; Rpt1: INI1 repeat 1; SChLAP1: Second chromosome locus associated with prostate-1; SUC2: Sucrose invertase; SWI/SNF: Switching defective/sucrose nonfermenting; Zip: Leucine zipper
\end{abstract}

\section{Acknowledgments}

Not applicable.

\section{Funding}

This work was supported by the National Natural Science Foundation of China (81372907, 81301757, 81472531, 81402009, 81572787 and 81672683), the Natural Science Foundation of Hunan Province (14JJ1010 and 2015JJ1022), the Mittal Innovation Foundation of Central South University (MX2016442 and 15MX46), and the Fundamental Research Funds for Central Universities of Central South University (2016zzts478).

\section{Availability of data and materials}

Not applicable.

\section{Authors' contributions}

$W X$ and $Z Z$ designed and revised the manuscript. $Y T$ wrote the manuscript and drew figures. JW, YL, PZ, CF, YW, XL and FX collected the related paper and created the tables. $X \mathrm{~L}, \mathrm{GL}$ participated in the design of the review. All the authors read and approved the final version of the review.

\section{Competing interests}

The authors declare that they have no competing interests.

\section{Consent for publication}

Not applicable.

\section{Ethics approval and consent to participate}

Not applicable.

\section{Author details}

${ }^{1}$ The Key Laboratory of Carcinogenesis of the Chinese Ministry of Health, Xiangya Hospital, Central South University, Changsha, Hunan, China. ${ }^{2}$ The Key Laboratory of Carcinogenesis and Cancer Invasion of the Chinese Ministry of Education, Cancer Research Institute, Central South University, Changsha, Hunan, China. ${ }^{3}$ Hunan Key Laboratory of Nonresolving Inflammation and Cancer, Disease Genome Research Center, The Third Xiangya Hospital, Central South University, Changsha, Hunan, China. ${ }^{4}$ School of Information Science and Engineering, Central South University, Changsha, Hunan, China.
Received: 10 August 2016 Accepted: 6 February 2017

Published online: 17 February 2017

\section{References}

1. Farrants AK. Chromatin remodelling and actin organisation. FEBS Lett. 2008;582:2041-50.

2. Clapier CR, Cairns BR. The biology of chromatin remodeling complexes. Annu Rev Biochem. 2009;78:273-304.

3. Kasten MM, Clapier CR, Cairns BR. SnapShot: chromatin remodeling: SWI/ SNF. Cell. 2011;144:e310-1.

4. Reisman D, Glaros S, Thompson EA. The SWI/SNF complex and cancer. Oncogene. 2009;28:1653-68.

5. Hohmann AF, Vakoc CR. A rationale to target the SWI/SNF complex for cancer therapy. Trends Genet. 2014;30:356-63.

6. Gong Z, Zhang S, Zhang W, Huang H, Li Q, Deng H, et al. Long non-coding RNAs in cancer. Sci China Life Sci. 2012;55:1120-4.

7. Lian Y, Li XY, Tang YY, Yang LT, Li XL, Xiong W, et al. Long Non-coding RNAs Function as Competing Endogenous RNAs to Regulate Cancer Progression. Prog Biochem Biophys. 2016:43:219-25.

8. $\quad$ Gong Z, Zhang S, Zeng Z, Wu H, Yang Q, Xiong F, et al. LOC401317, a p53regulated long non-coding RNA, inhibits cell proliferation and induces apoptosis in the nasopharyngeal carcinoma cell line HNE2. Plos One. 2014;9:e110674.

9. Wang Y, Mo Y, Gong Z, Yang X, Yang M, Zhang S, et al. Circular RNAs in human cancer. Mol Cancer. 2017;16:25.

10. Yu J, Liu Y, Guo C, Zhang S, Gong Z, Tang Y, et al. Upregulated long noncoding RNA LINC00152 expression is associated with progression and poor prognosis of tongue squamous cell carcinoma. J Cancer. 2017;8:523-30.

11. Yang L, Tang Y, He Y, Wang Y, Lian Y, Xiong F, et al. High Expression of LINC01420 indicates an unfavorable prognosis and modulates cell migration and invasion in nasopharyngeal carcinoma. J Cancer. 2017;8:97-103.

12. Bo H, Gong Z, Zhang W, Li X, Zeng Y, Liao Q, et al. Upregulated long noncoding RNA AFAP1-AS1 expression is associated with progression and poor prognosis of nasopharyngeal carcinoma. Oncotarget. 2015;6:20404-18.

13. Zeng Z, Bo H, Gong Z, Lian Y, Li X, Li X, et al. AFAP1-AS1, a long noncoding RNA upregulated in lung cancer and promotes invasion and metastasis. Tumour Biol. 2016;37:729-37.

14. Wang $Y$, Xue D, Li Y, Pan X, Zhang $X$, Kuang B, et al. The long noncoding RNA MALAT-1 is a novel biomarker in various cancers: a meta-analysis based on the GEO database and literature. J Cancer. 2016;7:991-1001.

15. He B, Li W, Wu Y, Wei F, Gong Z, Bo H, et al. Epstein-Barr virus-encoded miR-BART6-3p inhibits cancer cell metastasis and invasion by targeting long non-coding RNA LOC553103. Cell Death Dis. 2016;7:e2353.

16. Gong Z, Yang Q, Zeng Z, Zhang W, Li X, Zu X, et al. An integrative transcriptomic analysis reveals p53 regulated miRNA, mRNA, and IncRNA networks in nasopharyngeal carcinoma. Tumour Biol. 2016;37:3683-95.

17. Carlson M, Osmond BC, Botstein D. Mutants of yeast defective in sucrose utilization. Genetics. 1981;98:25-40.

18. Neigeborn L, Carlson M. Genes affecting the regulation of SUC2 gene expression by glucose repression in Saccharomyces cerevisiae. Genetics. 1984;108:845-58.

19. Peterson $C L$, Dingwall A, Scott MP. Five SWI/SNF gene products are components of a large multisubunit complex required for transcriptional enhancement. Proc Natl Acad Sci U S A. 1994;91:2905-8.

20. Muchardt C, Yaniv M. ATP-dependent chromatin remodelling: SWI/SNF and Co. are on the job. J Mol Biol. 1999;293:187-98.

21. Smith-Roe SL, Nakamura J, Holley D, Chastain 2nd PD, Rosson GB, Simpson DA et al. SWI/SNF complexes are required for full activation of the DNA-damage response. Oncotarget. 2015;6:732-45.

22. Wang X, Haswell JR, Roberts CW. Molecular pathways: SWI/SNF (BAF) complexes are frequently mutated in cancer-mechanisms and potential therapeutic insights. Clin Cancer Res. 2014;20:21-7.

23. Phelan ML, Sif S, Narlikar GJ, Kingston RE. Reconstitution of a core chromatin remodeling complex from SWI/SNF subunits. Mol Cell. 1999;3:247-53.

24. Xu K, Xiong W, Zhou M, Wang H, Yang J, Li X, et al. Integrating ChIPsequencing and digital gene expression profiling to identify BRD7 downstream genes and construct their regulating network. Mol Cell Biochem. 2016;411:57-71.

25. Peterson $\mathrm{CL}$, Workman $\mathrm{JL}$. Promoter targeting and chromatin remodeling by the SWI/SNF complex. Curr Opin Genet Dev. 2000;10:187-92.

26. Wilson CJ, Chao DM, Imbalzano AN, Schnitzler GR, Kingston RE, Young RA. RNA polymerase II holoenzyme contains SWI/SNF regulators involved in chromatin remodeling. Cell. 1996;84:235-44. 
27. Owen-Hughes T, Utley RT, Cote J, Peterson CL, Workman JL. Persistent site-specific remodeling of a nucleosome array by transient action of the SWI/SNF complex. Science. 1996;273:513-6.

28. Gangaraju VK, Bartholomew B. Mechanisms of ATP dependent chromatin remodeling. Mutat Res. 2007;618:3-17.

29. Zhang B, Chambers KJ, Faller DV, Wang S. Reprogramming of the SWI/SNF complex for co-activation or co-repression in prohibitin-mediated estrogen receptor regulation. Oncogene. 2007;26:7153-7.

30. Jiang C, Pugh BF. Nucleosome positioning and gene regulation: advances through genomics. Nat Rev Genet. 2009;10:161-72.

31. Hargreaves DC, Crabtree GR. ATP-dependent chromatin remodeling: genetics, genomics and mechanisms. Cell Res. 2011;21:396-420

32. Han P, Chang CP. Long non-coding RNA and chromatin remodeling. RNA Biol. 2015;12:1094-8.

33. Prensner JR, lyer MK, Sahu A, Asangani IA, Cao Q, Patel L, et al. The long noncoding RNA SChLAP1 promotes aggressive prostate cancer and antagonizes the SWI/SNF complex. Nat Genet. 2013;45:1392-8.

34. Lee RS, Roberts CWM. Linking the SWI/SNF complex to prostate cancer. Nat Genet. 2013;45:1268-9.

35. Wang $X$, Gong $Y$, Jin B, Wu C, Yang J, Wang L, et al. Long non-coding RNA urothelial carcinoma associated 1 induces cell replication by inhibiting BRG1 in 5637 cells. Oncol Rep. 2014;32:1281-90.

36. Gao C, Zhang J, Wang Q, Ren C. Overexpression of IncRNA NEAT1 mitigates multidrug resistance by inhibiting $A B C G 2$ in leukemia. Oncol Lett. 2016:12:1051-7.

37. Song Y, Li X, Zeng Z, Li Q, Gong Z, Liao Q, et al. Epstein-Barr virus encoded miR-BART11 promotes inflammation-induced carcinogenesis by targeting FOXP1. Oncotarget. 2016;7:36783-99.

38. Sun C, Li S, Zhang F, Xi Y, Wang L, Bi Y, et al. Long non-coding RNA NEAT1 promotes non-small cell lung cancer progression through regulation of miR-377-3p-E2F3 pathway. Oncotarget. 2016;7:51784-814.

39. Ke H, Zhao L, Feng X, Xu H, Zou L, Yang Q, et al. NEAT1 is Required for Survival of Breast Cancer Cells Through FUS and miR-548. Gene Regul Syst Biol. 2016;10:11-7.

40. Fu JW, Kong Y, Sun X. Long noncoding RNA NEAT1 is an unfavorable prognostic factor and regulates migration and invasion in gastric cancer. J Cancer Res Clin Oncol. 2016;142:1571-9.

41. Kawaguchi T, Tanigawa A, Naganuma T, Ohkawa Y, Souquere S, Pierron G, et al. SWI/SNF chromatin-remodeling complexes function in noncoding RNA-dependent assembly of nuclear bodies. Proc Natl Acad Sci U S A. 2015;112:4304-9.

42. Adriaens C, Standaert L, Barra J, Latil M, Verfaillie A, Kalev P, et al. p53 induces formation of NEAT1 IncRNA-containing paraspeckles that modulate replication stress response and chemosensitivity. Nat Med. 2016;22:861-8.

43. Cajigas I, Leib DE, Cochrane J, Luo H, Swyter KR, Chen S, et al. Evf2 IncRNA/ BRG1/DLX1 interactions reveal RNA-dependent inhibition of chromatin remodeling. Development. 2015;142:2641-52.

44. Tantai J, Hu D, Yang Y, Geng J. Combined identification of long non-coding RNA XIST and HIF1A-AS1 in serum as an effective screening for non-small cell lung cancer. Int J Clin Exp Pathol. 2015;8:7887-95.

45. Wang $S$, Zhang $X$, Yuan $Y$, Tan $M$, Zhang $L$, Xue $X$, et al. BRG1 expression is increased in thoracic aortic aneurysms and regulates proliferation and apoptosis of vascular smooth muscle cells through the long non-coding RNA HIF1A-AS1 in vitro. Eur J Cardiothorac Surg. 2015;47:439-46.

46. Wang $Y$, He L, Du Y, Zhu P, Huang G, Luo J, et al. The long noncoding RNA IncTCF7 promotes self-renewal of human liver cancer stem cells through activation of Wnt signaling. Cell Stem Cell. 2015;16:413-25.

47. Liang F, Li Q, Li X, Li Z, Gong Z, Deng H, et al. TSC22D2 interacts with PKM2 and inhibits cell growth in colorectal cancer. Int J Oncol. 2016;49:1046-56.

48. Hu G, Gong AY, Wang Y, Ma S, Chen X, Chen J, et al. LincRNA-Cox2 promotes late inflammatory gene transcription in macrophages through modulating SWI/SNF-mediated chromatin remodeling. J Immunol. 2016;196:2799-808.

49. Wang L, Zhao Z, Meyer MB, Saha S, Yu M, Guo A, et al. CARM1 methylates chromatin remodeling factor BAF155 to enhance tumor progression and metastasis. Cancer Cell. 2014;25:21-36.

50. Cheng SW, Davies KP, Yung E, Beltran RJ, Yu J, Kalpana GV. c-MYC interacts with INI1/hSNF5 and requires the SWI/SNF complex for transactivation function. Nat Genet. 1999:22:102-5.
51. Nagl Jr NG, Zweitzig DR, Thimmapaya B, Beck Jr GR, Moran E. The c-myc gene is a direct target of mammalian SWI/SNF-related complexes during differentiation-associated cell cycle arrest. Cancer Res. 2006;66:1289-93.

52. Shi J, Whyte WA, Zepeda-Mendoza CJ, Milazzo JP, Shen C, Roe JS, et al. Role of SWI/SNF in acute leukemia maintenance and enhancer-mediated Myc regulation. Genes Dev. 2013;27:2648-62.

53. Thomas LR, Wang Q, Grieb BC, Phan J, Foshage AM, Sun Q, et al. Interaction with WDR5 promotes target gene recognition and tumorigenesis by MYC. Mol Cell. 2015;58:440-52

54. Romero OA, Torres-Diz M, Pros E, Savola S, Gomez A, Moran S, et al. MAX inactivation in small cell lung cancer disrupts MYC-SWI/SNF programs and is synthetic lethal with BRG1. Cancer Discov. 2014;4:292-303.

55. Li Q, Chen P, Zeng Z, Liang F, Song Y, Xiong F, et al. Yeast two-hybrid screening identified WDR77 as a novel interacting partner of TSC22D2. Tumour Biol. 2016:37:12503-12

56. Fischer B, Sandmann T, Horn T, Billmann M, Chaudhary V, Huber W, et al. A map of directional genetic interactions in a metazoan cell. Elife. 2015;4:e05464.

57. Medina PP, Romero OA, Kohno T, Montuenga LM, Pio R, Yokota J, et al. Frequent BRG1/SMARCA4-inactivating mutations in human lung cancer cell lines. Hum Mutat. 2008;29:617-22.

58. Young NP, Jacks T. Tissue-specific p19Arf regulation dictates the response to oncogenic K-ras. Proc Natl Acad Sci U S A. 2010;107:10184-9.

59. von Figura G, Fukuda A, Roy N, Liku ME, Morris Iv JP, Kim GE, et al. The chromatin regulator Brg1 suppresses formation of intraductal papillary mucinous neoplasm and pancreatic ductal adenocarcinoma. Nat Cell Biol. 2014;16:255-67.

\section{Submit your next manuscript to BioMed Central and we will help you at every step:}

- We accept pre-submission inquiries

- Our selector tool helps you to find the most relevant journal

- We provide round the clock customer support

- Convenient online submission

- Thorough peer review

- Inclusion in PubMed and all major indexing services

- Maximum visibility for your research

Submit your manuscript at www.biomedcentral.com/submit
) Biomed Central 